\title{
La formación del bibliotecario en México: 1924, una propuesta de formación integral en el marco de la biblioteca moderna
}

\author{
Martha AliciaAñorVe Guillén \\ CentroUniversitariodeInvestigacionesBibliotecológicas \\ de la UNAM, 04510, Mé xi co D.F., Tel:56-23-03-65 \\ E-mail:anorve@servidor.unam.mx
}

\begin{abstract}
RESUMEN
Elpresenteartículopresentauna propues tadeforma ciónin tegralformulada por Jua na Man ri que de Lara des de Nue va York al pe rio do que se ha con si derado como de florecimiento de las bibliotecas en México, que encabezara José Vasconcelos, fundador de la Secretaría de Educación Pública, SEP en 1921 y que la autoramencionadapresentara a Jaime Torres Bodet, en tonces jefedelDepartamen todeBibliotecas delcitadominis terio. Dichapropues ta se des ta ca como una apor ta ción im por tan te, en aquel mo men to, para con tribuir a que las bibliotecas y los bibliotecarios jugaran su papel en la aspirada democratización dellibroylalec tu ra enla sociedad mexicana,yes considerada por la au to ra del pre sen te do cu men to como el fun da men to de los pla nes de estudio de bibliotecología en México durante el siglo XX.

Pa la bras Clave: For ma ción profe sional, Mé xi co, His to ria de la biblio te co lo gía.
\end{abstract}

\section{LIBRARY FORMATION IN MEXICO: 1924, AN INTEGRAL PROPOSAL WITHIN THE MODERN LIBRARY MARTHAAli CIA AÑORVE-GUILlÉN}

\begin{abstract}
A proposal for the integral formation of librarians is formulated by Juana Manrique de Lara to José Vasconcelos, founder of the Public Education Ministry in 1921, but Manrique de Lara also shows his plan to Jaime Torres Bodet, then Chief of the Li brary De part ment of the min is try. The sug ges tion is made from New York during the pe riod con sid ered as that of the blos soming for libraries in Mexico and stands out as an important contribution search ing that li brar ies and li brari ans played a role in the de moc ra ti za tion of books and reading in the Mexi can so ci ety. The pro posal is con sid ered by the authoress of this paper as the basis for the library studies of Mexico during the XX century.

Keywords: Pro fes sio nal, Mé xi co, His tory of the librarianship.
\end{abstract}




\section{INTRODUCCIÓN}

$\mathbf{E}$ nel marco de las in quie tu des y pro pues tas para la for ma ción de biblio te ca rios a partir del siglo XIX mexicano, el presente trabajo presenta y ubica el planteamiento de lo que denominaremos la concepción integral para la formación del bibliotecario comoelsus ten to dela edu cación bibliotecológica mexicanadelsigloXX. Este enfoque le fue señalado, entre 1923 y 1924, a Jaime Torres Bodet por Juana Man rique deLara du ran te su es tan cia de es tu dios en Nue va York, comoidóneopara la formación de los bibliotecarios mexicanos.

TorresBodeteraen tonces Jefe delDepartamen todeBibliotecas delaSecretaríade EducaciónPública(SEP) dependen ciaqueosten tabaelliderazgodeldesarrollobibliotecariodel paísduran tela que se ha de no mina dolaépoca deorodelas biblio tecasyel libro, lide ra da por el brillan tein telectual mexi ca noJoséVasconcelos, quien fue fundador de laSEPy suminis trodes de me dia dos de 1921 has tame dia dosde 1924, y quien en suproyectoeducativoycul tu ral colocóal mis monivelycomoactoresin teractuantes a la edu ca ción, el arte y el libro. Por su parte Jua na Man ri que de Lara, egre sa daen 1917 de laprimerageneraciónquese formóen laprimera es cueladebibliotecariosyarchivistas (Escuela Nacional de Bibliotecarios y Archiveros) fue contratada en 1922 por el DepartamentodeBiblio tecasdela SEPpara dirigirenla ciudad deMéxicolabibliotecapú blicapopular “Amado Nervo".Jua nase en con tra ba en aquellos años (1923-1924)rea lizandoestudiosdebiblioteconomíaenlaescueladebibliotecarios anexaalaBiblioteca PúblicadeNueva York, todavezqueporsu des ta cada ac tua ción como res pon sable de labiblioteca "AmadoNervo" había sidocomisionada porla SEP pararealizares tudios debiblioteconomíaenlaciudaddeNuevaYork.

Desde nuestro punto de vista la propuesta de formación integral hecha desde Nue va York porMan rique de Lara se sus ten ta ba en el con cep to mo derno de bi blio teca y de bibliotecario, enriquecía y rebasaba el enfoque reduccionista sobre la forma ción téc nica en biblio te co no mía con el que se aten día la for ma ción de biblio teca rios en México, y se aproximaba al perfil de un bibliotecario más erudito, con forma ción técnicayprepara do parala pres ta ción de servicios. Man rique deLaraproponía que a la formación del bibliotecario que se llevaba en México, basada fundamentalmente enlacataloga ciónylacla sifica ción, seincorporaranlasasignaturas de encabezamientos de materia, selección y consulta, que se prepara al bibliotecario para ela borarresú menes de li brosy revis tas que orien ta ranallec torenlaselección de suslec tu ras, y que el biblio te ca rio de biblio te cas pú blicas asu mie ra las fun cio nes de edu ca dor, pro mo tor so cial y fue ra él mis mo un lec tor ac tivo para que pu die ra cons tituirse en un ver da de ro guía para la lec tu ra. En el con tex to de esta úl ti ma meta te nía sen ti do lapro pues ta de que se fa milia riza ra tan to con elco no ci mien todela producciónbibliohemerográficacomoconlasnecesidadesculturales, informativasydelecturadelacomunidad. 
A pe sarde que la pro pues ta he cha por Man ri que de Lara se ins pi ra ba en el plan de es tu dios que cur sa baen la es cuela de bibliote ca rios delabiblio te capú blica de Nueva York, he mos en con tra do que este he cho no fue asu mi do por ella de ma ne ra acrítica, al contrario, su pro pues ta te nía como te lón de fon do, y por tan to como sus ten to, la nueva concepción sobre la biblioteca y el nuevo perfil del bibliotecario, calificados amboscomomodernos.

\section{DELERU DITO AL BIBLIOTE CARIO CON FORMACIÓN EN CATALO GACIÓN YCLASIFICACIÓN}

En el siglodecimonónico, hombres como el historiadorCarlosMaríaBustamante considerabanqueel cargo debiblio te cariodejefedebiblioteca debíaserdesempeñado por" [...] un sabio; pero hom bre de bien que ame a las le tras tan to como a la pa tria y que vivapersuadidodequelaRepública serátan tomásfelizyopulenta, cuantosmássabios abun denen ella, y que aquella debesersual má ciga para formarlos."1 De he cho éste era elperfilquesebuscabaparadirigirlanacienteBibliotecaNacionalPública.

Enlapro puesta decreación de laBiblio tecaNa cionalde al guna ma ne ra se amalga maban tan tolaidea de constituirlaenunservicio públicopara to das las clases so ciales comola de contarconunagran de y sun tuo sabiblio te caquepermi tie raqueMéxicose colocara al nivel de los principales países y capitales del mundo civilizado. Tal es lo queenten de mos cuan do Carlos Herre ro señala que Ma nuelEduardode Goros tiza, autor del proyecto y reglamento para la creación de esta biblioteca “[...]se presentó antelaJunta [serefierealaJuntaDirectivadeEnseñanza Pública, antecedentede la Direc ción de Enseñan za Pública] lle van do en sus ma nos el pro yec to de creación y regla mentodelaBibliotecaNacionaly Pública."'2Es probableque el conceptodelopúblico en ese mo men to se sus ten ta ra más bien en la idea de un servicio fi nan cia doporelEstado y en abrir las biblio te cas a un pú blico ma yor pero aún muy eli tiza do, lo cual es ta ba determinadosobretodoporlanaturalezadelascolecciones.

Desde sudiscu tidonacimien tola Biblio teca Nacio nalestuvoa cargode connota das personalidades como el dramaturgo Manuel Eduardo de Gorostiza, el Doctor JoséMa ría Benítez, elpolíticoJoséMaríaLa fraguay el políticoy periodis taJosé María Vigil. Entre las muchas cosas que hi cie ron es tos hom bres a fa vor de la Na cio nal sólomencionaremosalgunas ac cionesqueloscaracterizan, asíporejemploGorostiza y Benítez, quienes actuaron en diferentes momentos, mostraron tal convencimien to so brela exis ten cia de esta biblio te ca que pu sie ron a dis po si ción de ella tan to sus economíaspersonales como sus conocimientos. Como ejemplo de esto último

1 CartadeCarlos María Bus ta mante, dirigidaal PoderEjecu tivo, eldía27 de septiembre de 1829, citada en Carlos Herrero Bervera, "Las bibliotecas en México: 1821-1850", en Carmen Vázquez Mantecón, đ. al., LasbibliotecasmexicanasendsigoXIX, México,Secretaría de EducaciónPúblicaDirección GeneraldeBibliotecas, 1987, p. 27.

2 Ilid, p. 40. 
Go ros tiza ela bo ró el pro yec to para sucrea ción ${ }^{3}$ y Be ní tez “[...]dejo arre gla dos y dispuestos para el servi cio pú blico, los libros dela an ti gua biblio te ca dela Ca te dral,los delalibre ría del fina do doctor Arrillaga (delosje suitas) yla colec ción que pertene cía a JuanSuá rez y Na va rro y se com próa Ig na cio Cum pli do". ${ }^{4}$ Y a Vigil se debela cla si ficación degranparte delacolección.

Dado el bagaje cul tu ralyel amplioco no cimien todel mun docul turalycientífico que teníanes tos perso najes se daba porsen ta do que po díanocuparse de selec cio nar las co lec cio nes, de guiar al públi co a la lec tu ray de cla si fi cary ca ta lo gar, de ahíque el temade la forma ciónbiblio tecaria no fueraunapreocupación. Noobstantedebidoa los cargos que de manera simultánea desempeñaban algunos de estos directores, comoLa fragua, no siem prela biblio tecare cibíala aten ciónquenece sita bay otrasveces el arraigo a las éli tes le tra das, de las cua les pro ve nían es tos hom bres, ha cía que en la prác tica la se lec ción y el servicio se des via ra del idea rio de ser vi cio orien ta do h acia to dos los nive les de la so cie dad. A Vi gilpor ejem plo sele acu só ya en el México re volucionariodehabersidoelitistaenestossentidos. ${ }^{5}$

En ocasiones, ante la au senciadelos directivos, las bibliote cas que daban a cargo deperso nal me nos cali fica do pero de ma yor per ma nen cia en ellas, quienesin ten taban rea lizar ta reas que exigían co no ci mien tos dela bibliote cono mía, y en ge ne ralde la cul tu ra, que en rea lidad no po seían. Como ejem plo delas di ficul ta des que aca rrea ba el hechode que perso nal con las ca rac te rís ti cas an tes ci ta das ocu pa ra el cargo de una biblio te ca, se pue de se ña lar el de Joa quín Car do so, quien subs ti tu yó en la di rección de la Biblio te ca Na cio nal a La fra gua. Así, la ac tua ción y la “apor ta ción” de Car do so se re dujo, de acuer do con la cita quehace Herre ro de Gon zález Obre gón, a una asis ten cia pun tual tan to deél como de superso nal, asícomo a una “[...] ex qui si ta vigí lan cia [...] para evi tar pér di das de li bros y que los man cha sen ole arran ca ran ho jas los lectores." 6

A pesar del beneficio que podíasignificar el hecho de tener en la dirección de las bibliotecas a grandes eruditos, ya desde 1828, José María Irigoyen, político liberal quienen el con tex to delase culariza ción dela en señanzaylaculturalehabíapro puesto a la Cá ma ra de Dipu ta dos lacrea ción de la Biblio te ca Na cio nal, re co no cía, se gún men ciona Herrero, lanece sidad de que cuan domenos el manejodelos acervos es tur vieraalcuidadodeespecialistas, nodepolíticos. ${ }^{7}$

\section{Ilid}

4 Carmen Vázquez Man te cón. “Las bibliote cas en México 1850-1880”, en Carmen Vázquez Man te cón, đ. al. Lasbibliotecas... OpCit., p. 114.

5 Ma ría Lui sa de la To rre de Ote ro. "El alma de la bi blio te ca", enEllibroydpuedo, II, 2-3 (abril- mayo, 1923), p. 46.

6 Luis Gon zá lez Obre gón.LabibiotecaNacionaldeMéxicareseñahistónica1833-1910.México,Editora Nacional, 1910, p. 43-44. Citado en Carmen Vázquez Mantecón, đ. al., Las biblidezas..Op Cit, p.118.

7 Carlos Herrero Bervera. "Las biblio te cas en México: 1821-1850",en Carmen VázquezMan tecón, e. al, Lasbibliotecas..Op Cit, p. 24. 
Latomadeconciencia acercadelanecesidad deprepararpersonalparabibliotecas llevó a que en 1912, personajes como Francisco Gamoneda y Ezequiel A. Chávez dictarancursossobreclasificación. ${ }^{8}$

Desgraciadamente los cursos no llegaban a todos los bibliotecarios del país. En general los acervos y servicios de las bibliotecas estaban en manos de personal con baja es colaridad yen oca sio nes sinnin gu navo ca ción. Es tas situa cio nesinci dían seriamente en el funcionamien todelas bibliote caseimpedían que ellec tordis pusie ra delmaterialqueestababuscando.

Para re me diaresta si tua ción y elde sor den en que se en con tra ban las biblio te cas y archivos de la nación los constitucionalistas, ${ }^{9}$ fundaron en 1916 la primera Escuela NacionaldeBiblio tecarios y Archiveros, que seproponía formaralos bibliotecarios queelpaís requería. ${ }^{10}$

Para introducir la importancia que tenía la formación en biblioteconomía e ilustrar la situación que prevalecía en las biblio te cas, asícomolain sa tis fac ción dealgunos biblio te carios, Nicolás León, pro fesordeorganiza ciónde biblio tecas yarchivos delacitadaprimeraescuelanarrabaasusalumnoslosiguiente:

Vi si té ha más de vein te años la ca pi tal de uno de nues tros le ja nos Es ta dos y allíen con tré una rica biblio te ca, so bre todo en libros an tiguos, for ma da conres tos devarias quepertenecierona congregaciones monásticas. Fue el cen tro de mis de li cias aquella biblio te ca en la cual en con tréver da de ras preciosidades bibliográficasque con to [sic] la liberalidad, justo es decirlo, el bibliotecario me facilitó.

Desempeñaba ese cargo un individuo honorable pero de una ignorancia su pi na, pues su vida la ha bía pa sa do te jien do re bo zos y sir vien do de

8 Rosa María Fernán dez deZa mo ra. “Cro no logía biblio te ca ria mexica na 1900-1988”, enInvestiga dónBiblictecoógicaII, 3 (julio-diciembre, 1987), p. 49.

9 Cabe aco tar que a pe sar de las di fe ren cias de in te re ses po lí ticos, eco nó mi cos y so cia les en tre las dis tin tas faccionesinvolu cradas enla Revolu ción Mexica na de 1910 se coin cidía, aun que con di feren tes con cep cio nes, en la ne ce si dad de que la edu ca ción lle ga rá a to das las ca pas de la población mexica na. Asi mis mo es ne ce sa rio se ña lar que el cla mor por la edu ca ción po pular fue filtrado a la cau sa revolu ciona ria porlas facciones po pula res, aun queen realidad el movimien todenominado cons ti tu cio na lis ta fue el que lo gró con cre tar un pro yec to para el pro gre so na cio nale in cluir en su programalas deman das de edu ca ción po pularporlo quelosimpulso res de és tas tra ta ron de que el gobierno se ocu pa ra de po ner la lec tu ra y la es cri tu ra al al can ce de toda la po bla ción. El pro yec to biblio te ca rio fueli de ra do por Agus tín Loe ray Chá vez. Tam bién cabe re cor dar que el movimiento cons titucionalis taseiniciópropia men tepara derrocaral go biernousurpadorde Vic to ria no Huerta, lo cual se asen tó en el Plan de Gua da lu pe el 26 de mar zo de 1913, en ple na dé ca da re voluciona ria. Aunque este movimiento estuvo encabezado por el entonces gobernador del estado de Coahuila, Venus tia no Carranzaylidera do porhombres de cla ses sociales al tas, a élse unie ron otras facciones revolucionarias que buscaban también cambios sociales y econó mi cosque este movimientofueincorporando.

10 Agus tín Loe ra y Chá vez. "La pri me ra Es cue la N. de Biblio te ca rios y Archive ros", en Boletíndela BibliotecaNadionaldeMéxiø, II, 3 (ene ro, 1916),p. 122. Ci ta do en Mar tha Ali cia Añor ve Guil llén,El despertardelavocacónbiblioteconómicadeJuanaManniquedeL_ara(1897-1922)endmarcodelasinstituaiones biblidecanias desutiempa (Tesis de maestría en bibliotecología). México, División de Estudios de Posgrado,FacultaddeFiloso fíayLetras, UniversidadNacional AutónomadeMéxico, 2002, p. 35. 
es cribien te de úl ti ma cla se, en las ofi cinas del Go bierno. Servi cios de un amigo por mejo rarsu si tua ción pe cu nia ria ob tu vie ron para élel nom bramien to de biblio te ca rio, en con trán do se, como era na tu ral, en tal em pleo, completamente desorientado.

Vinieron los conflictos y las mortificaciones con los lectores (era él un hombre de ver güen za) y tam bién le ata có el splęn de sus telares. Un día, des pués que ya me tomó con fian za y vien do con cuán ta de fe ren cia el Señor Go berna dor de ese Es ta do me hablaba, me pidio [sic.] mein te re sa se ante el mis mo para que le die se otro em pleo, pues el (el bi blio te ca rio)sees taba enbruteciendbentrelos libros!! Estas fueron sus palabras.

Logrépa sara otra oficina y tan to él comola biblio te ca ga na ron cien to por uno. ${ }^{11}$

El otro caso se re fie re a un biblio te ca rio de una res pe ta ble Ins ti tu ción a quien un lector le pidió los periódicos de 1910 de un determinado diario. "Cómo, dijo éste, cree Ud.queno so tros guarda moslos perió dicos viejos; es tos se re ciben a dia rioyluego se rom pen outilizan para en vol tu ra. El mozo dela biblio te ca se aper cibió de aquello e in di có al bi blio te ca rio que allí exis tía la colec ción alu di da y en de mos tra ción de ellolapusoen manos delquela solicitaba.jQuéhermosotipodebibliotecario!"12

Amén delsa queo y de sor den que reina ba en algu nas bi blio te cas porlalu cha re volut cionaria, estos dos hechos ilustran la lamentable situación de los servicios bibliotecarios y delos bi blio te ca rios conla que Méxi co re cibía el si glo XX,la cualjus tifíca ba, más quesobradamen te, lain minentene ce sidad decrearuna for ma ción es pecíficaparalos trabaja do res delas biblio tecas, y que quienes abra za ran esta actividad tuvie ranlavocaciónyla prepara ción previa queles permitieraapropiarsetantodeuna formación técnicacomodeotraqueloshabilitaraparalaprestaciónadecuadadeservicios.

La fundación de la primera Escuela Nacional de Bibliotecarios y Archiveros fue planteada por los constitucionalistas y otros intelectuales que apoyaban su apertura como una condición indispensable para abrir las bibliotecas al servicio público. La apertura de biblio te cas para to dos los sec to res dela socie dad era tambiénunele men to importanteparadarcumplimientoalas demandaseducativasyculturalesdelpueblo.

La edu ca ción para el pue blo, ideal plan tea do des de el si glo XIX, fue asi mila do por las faccionesrevolucionariaseincorporadoalproyectodelconstitucionalis mor evolucionario, cuya finalidadprimeraeraalcanzarlalegalidad. ${ }^{13}$

Elplantea mien to biblio tecariodeldiscursocons titu cionalis ta fueabrirlas bibliotecas exis ten tes a todo el pú blico (la Na cio nal y las públicas es ta tales), y crear nuevas bibliotecasquefueranexpeditasyatendieranefectivamentealos diferentes sectores de la sociedad(niños, pa dres de familia, obreros, pro fe sionis tas, es pecialis tas). Noobstante que lo anterior implicaba la apertura de servicios bibliotecarios y la formación de

11 Ni co lás León. Biblioteconomáa;notasdlasleedionesaralesdaProfesarDr.NicolásLeénenlaEsuelaNadi nal deBibliotecariosyArdiveros México, An ti gua Im pren ta de Mur guía, 1918, p. 132.

12 Ibid

13 Hila rio Me dina. "In tro duc ción”, en DiariodeDebatesddCongesoConstituyente1916-1917.México, Ins ti tu to Na cio nal de Es tu dios His tó ri cos dela Revolu ción Mexicana, 1985, v.1,p.10. 
colecciones acordesconlos di fe ren tes grupos delaso cie dad, la forma ción del bibliotecarioseen caminó fun damentalmenteala catalogaciónyla cla sifica cióny, encierta me di da, a la ad mi nis tra ción de bi blio te cas. Si bien es cier to que con este en fo que se aten día elproblema cen traldela orga niza ción de colec ciones, también lo esquelimi tabalaparticipación delbiblio te ca rioenla con formación delascolec cionesy ele stablecimientodeunservicioefectivo.

Elencau ce de formaciónbibliote ca riacentra daenlaorganizacióndecolecciones yenlaadminis tración de biblio tecasque dó ex presado en el ob je tivode di chains titución, de manera que Agus tínLoera Chávez, fun da dordelaprimera escuela debiblio te ca rios y ar chi ve ros, se ña ló que la "Es cue la se crea con el fin de rea li zarla pre pa ración de bibliotecarios y archiveros idóneos, capacitados para llevar a cabo la reorganización y dirigir el funcionamiento de las Bibliotecas y los Archivos Oficiales." ${ }^{14}$ Esta declaración y las materias del plan de estudios (clasificación, catalogación, organización, conferencias de bibliología, traducción de francés, inglés y latín) ${ }^{15}$ nos marcan el divorcio en tre la for ma ción del biblio te ca rio y el plan tea mien to deabrirbibliote cas para to dos los sec to res dela so cie dad, lo que exigi ría que el bibliote ca rio fue raprepara dopara to marparte ac tiva en la selec cióny es pecial mentepara guiaralusuariohacialalectura.

Aunquepareceríapo sible quelasmirasparala formación debiblio tecarios yarchive ros se limi ta ran porquelas circunstan cias obliga ban a re te neral per so nal que yae stabaads critoa es tasins ti tu ciones yque, comoya he mos señala do, eran más bien personas con baja escolaridad y muchas veces sin vocación, observamos que las características delpersonalque trabajabaenlasbibliotecasno fueronlarazón para limitarla forma ciónalos aspectos deca taloga ción, cla sifica cióny ad minis tración. En la conformación del segundo plan de estudios de la citada Escuela, que se presentó como una opción profesional a los alumnos que egresaban de la escuela preparatoria, ${ }^{16}$ tam po co se con tem plaronlas asig na tu ras de selec ción y las rela tivas a la prepara ción para pres tarservicios, no obs tan te que se bus ca ba que la ca rre ra fue ra ele gida poregresadosdepreparatoria,cuyosantecedentesacadémicospermitíanin troducir materiascomoladeselecciónyladeserviciodeconsulta.

La au sen cia delas ma te rias de selec ción y con sul ta en la for ma ción del biblio te cariomexicano, noobstantequeés tas fue ranya obje to dela prepa racióndelbiblio tecarioen Es ta dosUnidos (en cu yos planes de es tu dio seins pi ró prin cipalmen te estapri mera escuela de bibliotecarios) nos permite observar una verdadera inconsistencia respec to delosideales de aten dercon colec ciones ade cua das alos diferen tes grupos delapoblación.

14 Agus tínLoe ray Chá vez. "La prime ra Es cue la N. De Biblio te ca rios y Archive ros", en Op Cit, p. 35.

15 Ibid p.36.

16 Nicolás León. Bibidteconomá...OpCit,, p. 141. 
Porotra partey es pe cial men te en la prime ra pro mo ción dela es cuela, los cons ti tut cionalis tasincurrieronen el errordecon siderarque el manejo dela cla sifica cióny la catalogaciónpodíaseralcanzadoporpersonas que sóloteníanlainstrucciónprimaria.

JuntoconIguínizconsideramosqueelobjetivode formarpersonalparaorganizar técnicamente las colec ciones ylos archivos y ad minis trares tas ins titu cio nes tampocopudo lo grarse alcien porcien to debidoalainclu sión depersonal con baja es colaridad y cul tu ra, que ade más se ins cri bió porobliga ción. ${ }^{17}$ El pro ble ma de la or ga ni zación técnica de las colecciones exige, ya desde el manejo de los instrumentos de catalogaciónyclasificación, unniveldees colaridaddefinitiva mentesuperior a la prima riay un buen nivelcul tu ral, so bre todo para asignarla cla sifica ción yencabezarlos documentos.

Si bien es cier to que du ran te el tiem po en que Vas con celos se de sem peñó como Secretario de Educa ción (1921-1924) pre vale cióla es tra te gia de en viaraalgu nos bi bliotecarios (evidentemente los menos pero los más preparados) a formarse en los Estados Unidos, ${ }^{18}$ la educación del conjunto de los bibliotecarios mexicanos se dio no a partir de una escuela de bibliotecarios sino mediante el dictado de cursos por conferencias una vez por semana. Estos cursos fueron dirigidos en un primer momen to al pú bli co en general conel fin de cap tarvo ca ciones y por tan to per so nal para trabajarenlas bibliotecas. ${ }^{19}$ En unsegun do mo men to, con un pro gra ma más de talla doy con el dicta do delas con ferencias dos ve ces porsemana, és tas se di rigieron " [...] a los empleados del Departamento que aspiraban al título de bibliotecario"20 y "[...]muy principalmente a los que tie nen a suin me dia to cui da do las bi blio te cas pút blicas." 21

Los con tenidos prin cipales de es tas con feren cias fue ron, comoen la Es cuela Na cional de Bibliotecarios y Archiveros, la catalogación y la clasificación no obstante que el ideal ma ni fies to en esta épo ca fue ra que las bi blio te cas y el li bro, al igual que la alfabetiza ción, la es cuelayelartenacional, se cons titu ye ran enverda deros colaboradores para lograr la anhela da re cons truc ción cul tu ral, educa tivay de progresoparae 1

17 JuanB.Iguíniz. “Apun tes parala his to ria dela en señanza dela biblio te conomía en Mé xico”, enBo letíndelaEsauelaNacional deBibliotecariosyArdivistasi II, 3-4 (enero.abril,1954), pp.13-17.

18 Entrelascuatropersonas dequienes tenemos noticiaquegozarondeestaoportunidades tu voJua na Man ri que de Lara, que fue por cier to quien cum plió con el anhe lo de mul ti pli car los be neficios de dicha estancia, difundir las idea de biblioteca moderna y quien, como veremos más adelante, pugnó por una nueva pre pa ra ción del biblio te ca rio que fue ra con gruen te con di choideal.

19 “Avi so", en Ellibroyed pueblo I, 2 (abril, 1922), p. 16. En di cho avi so se se ña la ba que el De par tamen todeBiblio te casinaugu raba "[...] un ciclode con fe rencias des tina das a divulgar conocimientos prácticos de bibliografía, biblioteconomía y bibliofilia" y se especificaba: "Las personas que de seen ins cribirse pue den ha cer lo des deluego en lain te ligen cia de que ha brá de prefe rírseles en el fu tu ropara cualquiernom bra mien to y que re cibi rán comocom pro ban te de sus es tu dios un di ploma de aptitud [...]"

20 "In formeleídodelDeparta men to en elCongresodeBibliotecarios, de Aus tin Texas", en Boleánde la SecretanáadeEducaciónPública,I, 3 (enero, 1923), p. 335.

21 LuzGarcía Núñez. "Memoria dela con fe rencia dela Ame ricanLibrary Associa tionylaS outh west LibraryAssociation”, en BoleándlaSecretańadeEducacónPública,I, 4, (1er. Se mes tre, 1923), p. 280. 
pueblo mexicano. El papel de la biblioteca en este contexto exigía del bibliotecario una participación di rec tay cons cien te conel público, ba sada enlos conocimien tosy metodologíasde la bi blio te co no mía y en una só li da cul tu ra gene ral, de ahí que en el Reglamen to deBiblio te cas se asen ta ra que para ocu parel pues to debiblio te ca rio se preferiría a aquellas personas que contaran con estudios de nivel medio superior, ${ }^{22}$ tradujeranalguna lengua moderna y se hubierandistinguido en al gu na rama del conocimien to hu ma no. En ten de mosque elperso nal que se con tra tó no siem prellena ba dichos re qui si tos y mu cho me nos con ta ba con el que alu día ala prepa ra ción en bi blioteconomía: "Saber los fundamentos de la clasificación de las ciencias y artes y demostrar prácticamente su conocimiento" y "Tener nociones sobre Bibliografía, Biblioteconomíaymateriasafines". 23

La aceptación de encargados de bibliotecas que no llenaban los requisitos indispensables para serlonos permi te en ten derque enla políticabiblio te caria que impulsaba $V$ as concelos, eran nue vamentelos hombres dele tras ${ }^{24}$ los que por síso los de finían la perspectiva de lectura y a quienes la SEP concedía el peso real para guiar al pueblohacia elmismoasun to queseaten día funda men talmen te median te Ellibroyd pudblórórga no de publicidad delaDirección Cen traldeBibliografía delDepartamento deBiblio te cas delaSEPy“"...]perió dicoex clusiva mentedes tinadoa orien taralpú blicoenla elec ciónylec tura delibros [...] in dican do quédebele er sey en dón de puede leerse."25 La con sul ta del ci ta do bo le tín exi gía en primerlu gar el do mi nio dela lec toescritura, cuando lo que se esperaba era lle gar a las ma sas de to dos los rin co nes del país, la que en su ma yo ría era anal fa be ta y en el mejor de los ca sos se mia nal fa be ta, lo quele dabaa las mayo rías muy poca po sibili dad de con sul taresta pu bli ca ciónyluego so li citarpores cri to las orien ta cio nes y ayu das que con res pec to al libroy la lec tura se ofrecíandes deelDepartamen to deBibliotecas. Sobrela soli citudpores crito se señala ba: "Si quie reus ted sa ber qué debeleer en cualquier or den del co no cimien to, es crí banos. Alrecibirsudirección,enviaremosaustedgratuitamenteestaRevista”. ${ }^{26}$

La realidadeducativa del país, dicho sea de paso, demandaba no obstante que en paralelo se trabajara en la alfabetización en español; que entre los recursos que se ofre cían en las biblio te cas se con ta ra con ma te riales de oralidad se cundaria, almenos en es pañol, y que el biblio te ca rio tu vie ra la pre pa ra ción quele pemitiera entenderla circunstan ciadelanal fabetayserunprovee dordirecto, oral, delainformación. Locual en tre otras co sas le exi gía el ma nejo delas obras de con sul ta y dela colección engeneral,

22 "Requisitos para ser bibliotecario", en Ellibroyd pueblo I, 6 (agos to, 1922), p. 47. En este do cumentosees tipulabaquesepreferiría alos as pirantes abibliotecariosquecontaranconpreparato ria onormal, tradujeranalgunalenguamodernaysehubierandis tinguidoenalgunarama de del co nocimientohumano.

23 Ibid

24 Ma ría Lui sa de la To rre de Ote ro. "El alma de la bi blio te ca", en Op Cit,p. p. 46. Esta au to ra asu mía que to ca ba "a los que más sa ben" la elec ción de las obras para las bi blio te cas".

25 "Invitación al pueblo y a la pren sa", en El libroye puddo, I, 1 (marzo, 1922), p. 1.

26 En ElLibroyd puebloI, 1 (marzo, 1922), p. 15. 
asícomodetécnicas decomunicación, prepara ción quedes gra ciada menteno recibía ensuformación.

Laselec ciónyadquisición de libros la realizabaen realidadelDepartamentode Bi bliote cas. Para encargarse de la selec cióny ad qui si ción de revis tas y perió dicos, este departamen to habíacrea dolaDirecciónCentraldeBibliografíayamencionada, que seen carga ría tam bién de la guía na cio nal parala lec tura, de di fun dira nivel na cio nal e in ternacionalla producción mexica na y enespañol,ydecon formarelcatálogogeneral de bibliotecas públicas del país “"...]con el fin de poder indicar en cualquier momen to ellu gar en que se en cuen tra cual quier obra." 27 A esta Di rec ción tam bién se le dele ga ba que se ocu pa ra de la for ma ción biblio grá fi ca del pú bli co y de do tara los bibliotecarios la cultura bibliotecaria "indispensable." 28 Este último calificativo nos muestra el papel elemental que también jugarían los bibliotecarios en la conformacióndeunasociedadlectora.

Sibien la omi sión dela forma ciónen selec ción pu die rajus ti ficar se porquelapropia SEPde ci día quéedi ta ba y com pra ba para las biblio te cas, lo cier to es quela ex pectativa en relación con el bibliotecario en esta época resultaba contradictoria, quizá debidoal en fren ta mien to en tre elideal y la rea lidad. Así, por un lado elám bi to re servadoalbiblio te ca rio era su propiabiblio te cayla comunidadala que ésta se dirigía, y al mis mo tiem po se le con fe ría un pa pel más bien co la te ral en la se lec ción de co lec ciones, otro deme dia na es ta tu ra en el fo men to dela lec tu ra, yun de sem peñocen tral en la organización técnica de las colecciones. A pesar de ello la capacitación que se le proporcionabaalfuturobibliotecario, aunadaasusgeneralmentebajosantecedentes aca démicosyculturales, nololepermitíandesempeñarconéxi to sus roles, aunlos relativosalaca talogaciónyclasificacióndelasobrasdesubiblioteca.

Portan to la or ga ni za ción téc ni ca de las colec cio nes fue con tem pla da comola tarea fundamental del bibliotecario. Con esta decisión se pensó favorecer no únicamen te a la biblio te ca que se te nía a cargo, sino también la con for ma ción del ca tálo go central que integraría la Dirección Central de Bibliografía dependiente del propio Departamento. Así, en el citado Reglamento se señalaba que era obligación de los res pon sa bles de bi blio te cas: "[...] llevara cabo o di rigirlas la bo res de ca ta lo gación" (en tién da se porellola organización técnica) yen con secuencia se manifes tabaque " [...] para la cla sifi ca ción delas bi blio te cas seharáuso del sis te ma de ci mal,empleándoseenla ca taloga ción tarje tas bibliográficas [...] portriplicado, envián dosealD epartamento de Bibliotecas de la Secretaría de Educación, un ejemplar para la formación del ca tálo go ge ne raldelDepar ta men to que lle va ráa cabola sec ción co rres pon diente". ${ }^{29}$ (laDirecciónCentraldeBibliografía).

27 "Invitación al pueblo y a la prensa", en Op Cit., p. 1.

28 Ibid

29 JoséVasconcelos. “ReglamentodelasBibliotecasPúblicas:disposicionesgenerales", en ElLibroy epueblo I, 4 (junio, 1922), p. 26. 
Sinembargolaparticipación delbibliote ca rioenla selecciónera tangencialaunen rela ción con las colec ciones quese asignaban a su pro pia biblio te ca, pues de acuerdo con elRegla men to delas Biblio te cas Públicas losins pec to res debibliote cas olos bibliotecarios tenían la atribución de proponerle a la SEP “[...]las obras que de ban adquirir se según las nece si da des de cadabiblio te ca." ${ }^{30}$ En cam bio sí era ma yor la par ticipa ción que se le pe día res pec to del fo men to a la lec tura, para lo cual tam po co sele preparaba. Parailus trarlas obliga ciones delbibliotecarioencuan toal tó picoan terior ele gi mos las que en rela ción a ello se le asignaban de acuer do con el ci ta do Re gla mento: "Ha cer una pro pa gan da real y efec tiva para lo grarel au men to de lec to res en la $\mathrm{Bi}$ blio te ca, en las fá bricas, talle res y es cue las del lu gar". En con tra mos que para ope rar lo anterior, en el mismo reglamento se indicaba también, aunque como actividades diferenciadas, que otras responsabilidades del biblio te ca rio eran las de " [...] or ganizar las con fe ren cias y lec tu ras" que se dic ta rían en la fies ta pú bli ca men sual que, de acuerdoconelmulticita do Reglamen to, de berían organizarseencadabibliotecapor lo me nos una vez al mes ade más de que los en car ga dos de bi blio te cas de be rían “" [...] pedira los direc to res delas Es cuelas que exis tan en ellu gar, quelleven a los alum nosa las salas de lectura, al efecto de acostumbrarlos desde pequeños a frecuentar estos centros de cul tura." "31 Cum plircones tas ta reas de man da ba la for ma ción co rres pondien te quele permi tie ra al biblio te ca rio ha cer suyo el conven cimien to del pa pelque jugabanlas bibliotecas enla de mocratiza ción dellibro,lalec tu rayel ac cesoala in formación,ylasmetodologíascorrespondientes.

Sin embargo, como ya he mos señala do, la forma ción seen fo có nueva men tey de manera especial a la catalogación y la clasificación. Esta decisión nos remite a una percepción res tringida delos alcances delbibliotecario enla forma ciónlectora. Además, los bajos sa la rios que se le ofre cían al per so nal bi blio te ca rio y que guarda ban rela ción con la po bre es co la ri dad y pre pa ra ción cul tu ral del per so nal que se te nía que contratar para las bibliotecas,podría ocultar el hecho de que subsistía la convicción deque eranlosintelec tua les, los hom bres dele tras compro me tidos con elrégimen, a quienes se consideraba realmente facultados para hacer la selección, para dirigir la lectura del pue blo mexica no y mos trarle al ex tran je rolo que en ma te ria de biblio tecas,yespecialmente, delibros, sehacíaenMéxico.

Lo anteriormente expuesto explica el hecho de que se haya minimizado el papel cen tral del bi blio te ca rio, y, por ende, el de la bi blio te ca en la de mo cra ti za ción del libro, en el de sa rrollo del gus to lec tor y en la guía para la lec tu ra, a pe sar de quein temacio nal men te se re co no cía que la biblio te ca era la ins ti tu ción por ex celen cia para encargarsededichoscometidosalestaren contactoinmediatoconlas comunidades. A pesardeestoúltimoy dequepara dójica mentese fundaron cien tos debibliotecas por todo elpaís, o al me nos se repar tie ran gran nú me ro de colec ciones, la pers pec tiva de lainfluenciadeunbibliotecariodebidamentepreparadotuvoqueabandonarse.

\section{Ibid}

31 Ibid 
Nuevamente se presentaba una inconsistencia entre las pretensiones del movimien to bi blio te cario, las fun ciones asigna das albiblio te ca rio en rela ción con la difusión delli bro y de la bi blio te ca en tre la co mu ni dad, y la for ma ción que se le ofre cía a éste. Como ya he mos se ñala do la ca pa ci ta ción se cen tró en en señarles “[...] el arte de catalogar y clasificar los libros para formar los diversos catálogos." 32 No obstante que ésta fue ra la for ma ción cen tral hay que re co no cer que como en la es cue la de biblio te ca rios de 1916 éstos no fue ronlos úni cos te mas que seimpartieron. Cabeseña larquela ca ta lo ga ción y la cla si fica ción se acom pa ña ron con te mas delabiblio gra fía como fue el re la tivo a "[...] el origen y des crip ción dellibro, ma te ria in te gral de las bibliotecas." 33 Asímismo se trataron otras materias de la biblioteconomía, por ejemplo, "[...-]lasnocionesin dis pen sables para organizaryhacerfuncionardebidamente esta cla sedeins titu ciones [las biblio tecas]." ${ }^{4}$ En esta úl ti ma ma te ria se to ca ban conte ni dos rela cio na dos conelpapeldelas biblio te cas en la an tigüe dad y enla épo caactual, los biblio te ca rios, los fon dos biblio grá ficos, la ad qui si ción de libros y el servicio alpúblico. Noobs tan tetodoellocon side ramos que nueva men te seperdió de vis tael he cho de que no era po si ble lo grar ni si quie ra el ob je ti vo de for mar ca ta lo ga do res y clasifica dores sielpersonalquese preparabanocontabaalmenos con es tudios den ivelmediosuperioryconuninterésverdaderoporel trabajoenbibliotecas.

Ade más podemos afirmarque la pre pa ra ción para el fo men to dela lec tu raylaguía ha cia la mis ma, al me nos la ne ce sa ria para in fluir en la co mu ni dad que aten día la biblioteca, es tuvoausente, y tambiénloes tuvolaque hubiera permitidoqueelbibliotecario participara activamente y conjuntamente con otros grupos en la selección de colecciones. Enconsecuencialaformación seremitióefectivamentealayacitadadotación "indispensable" de cultura bibliotecaria, que le ofrecía albiblio te ca riola DirecciónCentraldeBibliogra fía,yporesoloscursos queimpartióIguínizrecibie ronel calificativodecursoselementalesdebibliografíaybiblioteconomía.

La rela tividad de esta me di da de forma ción, aun en lo con cer nien te a la or ga ni za ción de las colecciones, se encuentra manifiesta en el señalamiento que hizo Esperanza ValázquezBringas cuando ocu pólaDirec cióndelDepartamen to de Bibliotecas "[...] solamente había tres bibliotecas catalogadas", 35 situación que a juicio de Velázquez Bringas se debía tanto a negligencia como a falta de conocimientos por partedelpersonaldebibliotecas. ${ }^{36}$

32 Juan B. Iguíniz. "Cla ses de biblio lo gía y bi blio te co no mía”, en BoletíndelaSecretańadeEducadónPú blica, I, 4 (1er semestre, 1923), p.295.

33 Ihid

34 Ibid

35 "In formeque rin delaSeñorita Es peranza VelázquezBringas, Jefe delDepartamen tode Biblio te cas al Doc tor José Ma nuel Ruiz Casau ranc, Se cre ta rio de Edu ca ción Pública, acer ca delaslabores de sa rro lla das du ran te el mes de ene ro", en BoleándelaSecetariadeEducadónPúblicą III, 9 (fe bre ro, 1925), pp. 137-142. 


\section{EL CONCEPTO DE BIBLIO TE CAYDE BIBLIOTECARIO MO DERNO, SUSTEN TO DE LAPRO PUESTADE FOR MACIÓN DE JUANA MANRIQUE DE LARA}

A pe sarde quela pre pa ra ción previa ne ce sa ria para ac ce der a una for ma ción en bi blioteconomía no fuera se ñala da como in dis pen sable por Man ri que deLara, no obs tan te que esto lo exi gie ra la es cue la de bi blio te ca rios de Nue va York, de la cual ella tomara supropuesta, presentamos a con tinuaciónla propues taquehemosdenominado de "formaciónintegral"delaquefueraportadoraJuanaManriquedeLara.

Man rique deLaracons ciente deque el res tringidoen foqueque se dabaen México a la prepa ración delbiblio te cariore du cía la po sibilidad de ac tua ción deés tosy de la biblioteca en el proceso de democratización de la lectura, del libro y de la información, propusoalDepartamen to deBiblio te cas delaSEP, que era en ton ceslains tan cia más po de ro sa en el de sa rro llo bi blio te ca rio nacio nal y por tan to la que antela au sencia de una es cuela de biblio te ca rios se ocu pa ba dela prepa ra ción de personal para desempeñarse en biblioteca, un nuevo enfoque de formación congruente con el concep to de no mina doporella "biblio te cay bibliote ca rio moderno" alcualtra tabande responderlosplanesdeestudioenbiblioteconomíaenlos EstadosUnidos.

En contraposición al " [...] antiguo y errado concepto de las bibliotecas que las consideraba como un simple hacinamiento de libros más o me nos arre gla dos, y cuyos precio sosvolúmenesllegabanamanos delosescasísimossolicitantesdespuésde infinidadderequisitosycortapisas"Juanapresentaelnuevoconceptodebibliote cay enconsecuencia de biblio te cario, que de no mina "la biblio te cayelbiblio teca riomoderno". 37

Sibien de fine a la biblio te ca mo derna como aquellains ta la da en un edi ficio es pacio so, atrac tivo, lim pio, ilu mina do, bien ubi ca do y que cuen ta con los es pa cios y servicios que facilitan la lectura, (entre ellos el servicio de fotografía de libros y documen tos), sus ten ta el pa pel so cial de la bi blio te ca mo der na en la premisa de que " [...] los li bros es tán en la biblio te ca para que el pú bli colos leay [portan to, señala] se debe fomentara toda cos ta el amoryla fa cilidad para sulectura" 38 y es pe cificala importanciaque tiene es pecial men telabiblio te ca po pularcomoguía a la lectu ra decalidady en la formacióndelgustoporlalectura.

Bajoes tos principios, Jua naseñala que la bibliote ca moderna debe con tarconacervosbibliográficos “ [...] escogidossegún las ne ce si da des dela re gión, el gra do deintelec tualidaddesus lec to res ylos di fe rentes fines para [los que ha sido es ta ble cida]"y que esos acervos de ben es tararre gla dos " [...] con forme a las re glas téc ni cas de la bi blioteconomía", y en con secuen cia de ben con tar con ca tálogos de "fácil con sul ta”y proporcionarle a las personas todas las facilidades para acercarse al libro y a la información que

37 Juana Man riquedeLara, "Biblio te cas ybiblio te carios", enElLibroydPuedo III,3 (enero-marzo, 1924), pp. 33-35.

38 Ibid, p.35. 
requiere, en fun ción de esto úl ti mo les re cuer da a los bi blio te ca rios que sibien "la regla men ta ción y méto dos son ne ce sa rios para el or den dela bi blio te ca; [...] nun ca de benserunobstáculoparaque ellectorseacerqueconlibertadallibroquenecesite."39

Labibliote camodernasos tieneManrique deLara “[...] debeseruncentroeficienteygratuito dein forma ción tan to bibliográfica comogeneraly es tarsiempre en aptituddecontes tartodaclasede preguntasqueelpúblicolehaga." 40

En aras de fomentar el gus tolector, para Jua nala biblio te ca debe cons ti tuirse en centro de la vida de la comunidad, y ofrecerle por ejemplo conferencias, exposiciones, et céte ra, o bien los es pa cios para quela co mu nidad, in clui dos los sec to res de dicadosalosnego ciosy hasta losgru pos religio sos, organicenactivida des deestecorte o simplemente se reúnan para divertirse. Considera que la promoción del libro y la lectura es parte im por tan te del trabajo biblio te ca rio y quela im plementación de acciones paralograrestapromoción requie reimaginación práctica, alavezquedemandala bús que da de es tra te gias para ligarla bibliote ca con la vida de la co mu ni dad, ya la vida de la comunidad con la biblioteca. Por último Juana agrega que las bibliotecas pue den servirtam bién como cen tros de prác tica para los as pi ran tes abiblio teca rios. Así, con clu ye: “Todo esto, y po nien do al fren te a un biblio te ca rio com pe ten te, es lo queactualmenteconstituyelabibliotecaideal." ${ }^{\prime 1}$

Deesta forma, para Man rique deLara el biblio te ca rio es elele men to clave paralograrquela biblio te ca mo derna cum pla con la función so cial que tiene en comen da da. Talconvencimientolallevaaafirmar:

A pesarde to das las ven tajas de una bue na organiza ción ma te rialdelas bi bliotecas, puede decirse que el éxito o fracaso de ellas depende casi por completo del bibliotecario que posea. ${ }^{42}$

Dado que el “[...]biblio te ca rio es el alma de la biblio te ca" 43 y debe ser un guía por excelenciahacialalectura,ManriquedeLaraponeunénfasis especialenla formación (conocimientos, ac titu desyaptitudes) queelbiblio te cario re quiere paraju garsu papel preponderante, el que en términos más generales podríamos calificar como de democratizadordelalecturaydelabiblioteca.

Así, des pués demen cio narque existen es pecificida des en el per fily por tan to en la forma ción del bi blio te ca rio en fun ción de los di fe ren tes tipos debiblio te cas, señala queelbibliotecariomodernoes:

\footnotetext{
39 Ibid

40 Ilid, p. 34.

41 Ilid, p. 33.

42 Ibid, p. 34.

43 En una publicación de Jua na de 1922, en con tra mos que ella ha bía com pren di do ya que el papel cen tral de una biblio te ca (en este caso in fan til) era el que ju ga ba el biblio te ca rio, por lo que lo in troduceubicán dolocomo "elalmadeunabibliote ca".JuanaMan riquedeLara, "Biblio tecasinfantiles y juveniles”, en Biblosiv, 158, 159 (28 ene ro, 4 fe bre ro, 1922), p.15.
} 
[...]un pro fe sionis ta quenece sitahaceres tudios técnicos, se rios, sobresu profesión y no un simple guardador y mozo de libros como hasta hace poco se creía [...]. Apar te de esto, se le exi ge ante todo una bue na y ex tensacul tu rageneral, es píritude cor te sía y de servicio,imagina ción prác tica y entusiasmo por su trabajo. ${ }^{44}$

En rela ción con la forma ción y eles tre cho com promi so que elbiblio te ca rio debibliotecas públicas po pulares tieneeneldesarrollodela conductalecto ra Juana señala que:

En las bibliotecas populares, el bibliotecario tiene un amplio campo de trabajoy frecuen temente seconvertirá en un pro fesory enun trabaja dor so cial. Su prin cipaly más im portan te fun ción con sis tirá, ante todo, en poner en contacto al lector con el libro que necesite $[\ldots]^{45}$

Estrechamente relacionado con la formación del bibliotecariopara guiar a otros enlacul tu ray para desempeñareficien tementesulabordein formación, Jua nadesta calanecesidaddequeelpropiobibliotecarioseaunlector;así,señala:

Un buen bibliotecario deberá tener amor por los libros; pero no con la particularafición deun biblió ma noo deunbiblió filo [...], sinoquedeberá amarlos por lo que en sí valen como receptáculos que conservan para siem pre el fru to de los ce re bros privile gia dos. Este amor porlos li bros en tal for ma, lo con du cirán a leer mu cho, cosa in dis pen sa ble para un bi blio te ca rio, guía de otros en la cul tu ra, y cuya ins truc ción y edu ca ción in telectual constituye la base de su profesión. Esto mismo le servirá [...] en su labor de información que es una de sus importantes funciones. ${ }^{46}$

El concepto de biblioteca moderna y las ideas expresadas por Manrique de Lara acercadel co me tidodeésta, permitenen ten derlaimportancia de quela forma cióndel bibliotecariosesus ten teen "[...] una buenayex ten sa cul turage neral”, enun "[...] espíritu de cor te síay de servi cio” enima gi na ción prác ti ca y “[...] en tu sias mo por su tra bajo”. Asimis mopermiten dimen sionarelamplioy pro fun dosignifica do que para ella tiene, loquehadenominado“[...]loses tudiostécnicosenbiblioteconomía”. ${ }^{47}$

Para Jua na la for ma ción téc ni ca no se re du ce sólo aim par tirco no ci mien tos y habili da des para la or ga ni za ción téc ni ca delas colec cio nes y para la con se cuen te elabora ción y or ga niza ción de ca tálo gos, sino a lo graruna ver da de ra com pren sión del cometido socialdelabiblio tecay delas me to dologías bibliote conó micas quecon tribuyen a que el bi blio te ca rio sea un agen te ac ti vo en la selec ción delos acervos, unguía ha cia la lec tu ray, en tér mi nos más ge nerales, en al guien que permite ellogro de la democra tiza ción delalec tu ra y delain for ma ción para quela biblio te ca se con vier ta en elespacio porexcelen cia para que la co munidadencuen treeducación,in formacióny recreación.

44 Man rique de Lara. "Biblio te cas y bi blio te ca rios", en Op Cit, p.34.

45 Ibid

46 Ibid, p.35.

47 Ilid, pp. 34-35. 
Entendemos que para Manrique de Lara la formación en biblioteconomía (que entrega al bibliotecariotantoconocimientoscomohabilidades y actitudes para ayudarloaquelabiblio teca desempeñesuta reaenla de mocratizacióndelalec tu rayl a información) debe estarsoportada en una cul tu ra am pliay só li da, en una vo ca ción de servicio y en una búsqueda de actualización, ${ }^{48}$ amalgama que permite la formación delbibliotecario.

\section{3-1924, PRO PUEST AIN TE GRAL PARA LA FORMACIÓN DEL BIBLIOTECARIO}

Para Jua na, igual que para Dewey y para los cons ti tu cio na lis tas, era evi den te que el funcionamiento de las bibliotecas estaba estrechamente relacionado con la preparacióndelbibliotecarioyqueesteproyectoeraconsustancialaldesarrollobibliotecario.

A pesardeque el México ema na do dela re volu ción as pi ra ba a cons ti tuir bibliotecas modernas comprometidas con la democratización del libro, la lectura, la informa ción y la for ma ción del gus to lec tor, en el mo men to cum bre de fun da ción de bibliotecas en todo el país, la educación bibliotecaria, paradójicamente, no era un pro yec to cen tral, ni si quie ra la ca pa ci ta ción que sele propor cio na ba al biblio te cario eracongruenteconloscometidosatribuidosalabibliotecamoderna.

Bajolapretensión dequela for mación delbiblio te cariocorres pondie raala aspira ción revolu ciona ria delle varlalec tu ra y las biblio te cas al pue blo, que era congruente con el con cep to de Jua na Man rique de Lara so bre la biblio te ca y el bi blio te ca rio moderno, ella le pro pone a To rres Bo det una se rie de pro yec tos para me jo rar las con dicio nes de las biblio te cas de nues tro país, en tre las cua les es ta balacen traliza ción de la cata lo ga ciónyla clasifica ción, perole seña la que el proyec to cen tral quepermi tiría el funcionamiento de los otros debe ser, sobre todo, la formación de bibliotecarios, mismaqueleplanteadesdeunanuevaperspectiva.

Aun que la pro pues ta de Man ri que de Lara con te nía la no ción de avan zarha cia el restablecimientodeunaescueladebibliotecarios ${ }^{49}$, lo que convenien tementelepropo ne a To rres Bo det para es tar en el te rre no de lo po sible, es en riquecer con nue vas

48 Ibid Res pec to delas formas de ac tualiza ción Jua na señala lo siguien te: "Porúl timo, unbibliotecario progre sis ta pro cu ra rá es tar siem pre en con tac to con sus com pañe ros deprofe sión para ha cer in tercambio cons tan te deideas y no ti cias que au men ten su ha bilidad, asícomoleer publica ciones ylibros que se re fie ran a su pro fe sión para que se re nue ven sus co no ci mien tos y acre cien te su prestigio".

49 Jua na Man rique de Lara. "Pro gra ma dela Es cue la de Biblio te ca rios ane xa a la Biblio teca Pública de la ciu dad de Nue va York" en El libroyd pueblo II, 8-10 (oc tu bre-diciembre,1923),p.201.En este documento Juana le señalaba a Torres Bodet la idea de abrir una escuela de bibliotecarios cuan do le co men ta ba: "Mi obje to al en viar le nota de ta lla da de los es tu dios que es toy ha ciendoen esta es cue la es, en pri merlu gar, ha cer de su co no ci mien to la im por tan cia y ex ten sión de los es tudios y en se gui da, el de ha cerle un es que ma del cur so por en te ro con el fin de que pue da, en el fu turo, ser nos útil, ya sea que se es ta blez ca en Méxi co una Es cue la de Biblio te ca rios, o cuandomenos paraquesepuedandarcon fe ren cias so bre di fe ren tes ma terias bibliográ ficas, llevan do un plan determinado." 
materiaslacapacita ciónimpulsa dapor Vasconcelos, aunquedes denuestropuntode vis taloquereal menteseñalaba erala nece sidaddecon tarconun nuevoen foqueenla formacióndelbibliotecario:

El primero e indispensable paso que es necesario dar con el objeto de un mejoramien togeneraldelas condiciones presen tes del trabajodebibliote cas, es el de continuaryreforzarcondasesnuevaslasconferendiasdebiblioteconomáa que has ta aho ra di ri ge el se ñor Juan B. Iguí niz con el cuer po de bi blio te carios dela ciu dad de México.Las cla ses que se ría ne ce sa rioin tro du cir, se rían las deSelec ción deLibros, Encabezados deMa teriay Ad minis tración propiamente dicha, dando además, elemental información sobre sucesos mundialesydomésticos, serviciodeconsultaenbibliotecas, yalgunas pláti cas so bre las biblio te cas de di fe ren tes ti pos en los Es ta dos Unidos. Como consecuen cia de este trabajo de en señan zaven drála ne ce sidad de es tablecer el escalafón de los empleados de bibliotecas. ${ }^{50}$

Conelloproponía pa sarde una pers pec tivareduc cionis tadela formación técnica en bi blio te co no mía u una vi sión mu cho más amplia de ésta. En su pro pues ta tam bién rescataba la formación cultural general, el conocimiento de la dinámica internacional, na cio naly local, y la for ma ción para el servicio de con sul ta, aun que se ña la ba que estos conocimientos se impartieran en forma "elemental", seguramente pensando enlosantecedentes escolaresdelosempleadosdebibliotecas.

Lapers pectivain te gral, congruen te conel con cep to de bibliotecamoderna, laencontró Juana reflejada en el plan de estudios que cursaba en la citada escuela de bibliotecarios deNueva York, ${ }^{51}$ el cual in cluía ma te rias "nuevas" toda vez que el me dio bibliotecariomexicanonolashabíaconsideradohas taelmomentocomoasignaturas paralaformacióndelbibliotecario.

No hay que de jar de men cio nar que jun to a esta pro pues ta Jua na plan teóla de traduciryadap taralgu nos tex tos impor tan tes en biblio te co no mía que se usa ban enEs tados Unidos, ${ }^{52}$ einclusoella mis maes cribióenesemo mentoar tículos rela cionados conalgunas delas materiasqueproponía.Algunosdelosartículos quees cribiódes de Nueva Yorkserevisanenestedocumento.

Manrique de Lara estaba convencida de la importancia que tenían las "nuevas" materias para elfunciona mien to realdela bibliote ca mo derna, parala for ma ción del bibliotecario y por ello proporcionó como base para su impartición los programas queellacursabaenNueva York.

Jua na sos te nía que el biblio te ca rio de bía participaren la selec ción, porlo que afirmabaque:

50 Jua na Man rique de Lara. "Proyec to de re for mas e in tro duc ción de sis te mas de bi blio teconomía, segúnlos méto dos Norte America nos, enlas biblio te cas dela Re pública mexica na" en Ellibroyed puado, III,7-9, (julio-septiembre, 1924), p. 174.

51 NNC-RBML. The Colum bia Univer sity Rare Books \& Ma nus cript Li brary. Li brary School of the New York Public Library- Box 31 Jua na Man ri que de Lara 1923-1924. [Stu dent re cord].

52 Man ri que de Lara. "Pro yec to de re for mas..." en Op Cit, p.174. 
Una de las atribu cio nes del bi blio te ca rio con sis te en re cha zaro acep tarlos libros que sele pro pongan para ad qui si ción de la biblio te ca, y no sólo de berá re cha zar aquellos que por su in mo ra li dad o fal ta de mé ri to no de ban ser ad qui ridos, sino los que por su ca rác ter es pe cial no se adap ten a laín dole de la biblio te ca, ya sea porque son li bros ra ros o pre cio sos, en cuyo caso de ben serad qui ridos porlas biblio te cas-mu seos o porlas grandes bibliotecas, oya porque tra ten dema te rias cuya bibliogra fía sea muy ex ten sa en su es tableci miento o porque no haya probabilidad de ser solicitados. ${ }^{53}$

Así,paraayu daralos biblio te carios mexicanos, principalmente alos debibliotecas públicas populares a asumir su papel fundamental en la selección, escribió el articulo denomina do "La selec ción de libros paralas dife ren tes cla ses de biblio te cas y en especialparalas bibliotecas públicas" ${ }^{54}$ sus ten tan do su con te ni do en las re glas y en los conceptosdeMarySalomeCuttlerFairchild, biblio te caria norte americana queconsideraba que la fun ción de la biblio te ca mo derna “[...] es la de de sa rro llary en rique cerlavida hu manadela co mu nidadlle van doal pueblo [...] todos los libros que dehe cholepertenecen," 55 yquien sos teníala con cepción dela biblio te ca como me dioim por tan tísimo deeducaciónpopular. ${ }^{56}$

Aun que Man rique de Lara no es cribe en los años de 1923-1924 un artícu lo so bre el serviciode consulta, sos tiene quelaguía alalec tu raesuno delos principales co metidos de la bibliotecamoderna, y tam bién in di ca que la la bor de in for ma ción "[...] es una de las mas importantes funciones que debe realizar el bibliotecario." ${ }^{57}$ Comenta que el servicio exige habilidad para establecer una buena comunicación y el desarrollo de la ca pacidad de ob serva ción, estoúl ti mo paraperca tarse porejem plodel "gradodeintelectualidad" dellector, tannecesa rio paraguiarlo enlalecturay para proporcionarle información. Asimismoconsideraquelascualidadesanterioresdebenapoyarseen “[...]el es pí ritu de cor te síay deservi cio en bene ficiodel pú blico que acu da a la biblio teca", ${ }^{8}$ y que todas estas características deben acompañarse de una sólida cultura general y de há bi tos delec tu ra que sonim por tan tes en quie nes guían a otros en la cul tu ra y proporcionaninformación.

Juanaasu mequeelpapeldel biblio te ca rioenel servicioyla consul taalpúblicoes a talgradoimportantequecomenta:

53 Ibid

54 Jua na Man rique de Lara. "La se lec ción de libros para las di fe ren tes cla ses de biblio te cas y en es pecial para las bibliotecas públicas”, en El libroy d pudblo III, 10-12 (octubre-diciembre, 1924), pp.227-229.

55 Ibid p. 228.

56 Ibid

57 Juana Manrique de Lara. "Bibliotecas y bibliotecarios", en Op Cit, p. 35.

58 Ibid 
Se dice que es pre fe rible que una bi blio te ca esté téc ni ca men te mal arre gla da, pero que ten ga un bi blio te ca rio ama ble y bien dis pues to a ayu dar al pú blico en la busca de libros, que una cuya organización sea irreprochable, pero cuyo bibliotecario malhumora y áspero ahuyente a los lectores. ${ }^{59}$

De acuer do con el progra ma que se se guía en la es cuela de biblio te ca rios de Nue va York, es probablequeloqueJua naproponía que seimpartieraenla materiadeconsul ta era que se fun da men ta ra el pa pel del de parta men to de re fe ren cia o con sul ta en las bi blio tecas públicas, yqueseen seña ra elmanejodelosprincipales dicciona riosyenciclopediasconunadidácticaqueayudaraenlasolucióndeproblemas deinformación.

Entendemos que para el conocimiento, la orientación y la difusión de la lectura, Juana pug nó porque se for ma ra al biblio te cario enla ela bora ción de re súmenes delibrosy revistas, de ahíquesos tuvieronque:

Todo mo derno biblio te ca rio está obliga do a conocer has ta en sus menores de ta lles, el arte de la ano ta ción bi blio grá fi ca, y tam bién el de ha cer revis tas de li bros que pue dan lle varel se llo de su perso na li dad y una cla ra y justificada crítica de los libros que intenta juzgar. ${ }^{60}$

Por ello, y apoyándose en una expectativa de su tiempo,Juanase ñaló que“[...]el catálogo del porvenir será anotado", y agregaba que "[...]en algunas bibliotecas de Es ta dos Unidos se estálle van do a efec to eseideal." ${ }^{11}$ Su opi nión era “[...] que las simples lis tas de títulos al an tiguoes tilonocumplen ni con mu choconloque se es perade ellas", de ahíquein dica ra que "[...]las biblio tecas de be rán serlas primerasen in troducireste nuevo sis tema dein forma ción bibliográfica, en susbole ti nes, hojas volantes, anuncios, etc., asícomoensus lis tas es pecia les delibros so brede terminadoasun to,o paraciertosgruposdepersonas." 62

Conside rabaque "[...]el principalob je to dela ano ta ción es des pertarelin te rés sobre un li bro dado, con el ob je to de que el lec tor sien ta el de seo de le er lo, ya sea comprándolooconcurriendoalabiblioteca". .3

Plan teabaade más quelas bibliogra fías ano ta das son im portan tes no sóloparalos estudiosos e investigadores sino también para el trabajo técnico de los propios bibliotecarios. ${ }^{64}$

59 Ibid

60 Jua na Man ri que de Lara." Ano ta cio nes y re vis tas de li bros", enEllibroyd puebloIII, 4-6 (abril- junio, 1924), p. 89.

61 Ilid, p.92.

62 Ibid

63 Ibid, p.91.

64 Ibid, p.92. 
Para apo yarla for ma ción del biblio te ca rio en la ela bo ra ción de re sú menes Jua na escribeenesemomentoelartículo "Anotaciónyre vis tas delibros" siguien do funda mentalmentelosconceptos, losmétodosylas técnicas parala ano ta ción presentados porelbibliote cario británi coErnestSavage ${ }^{65}$ en su Manual ofdescriptiveannotationforli brarycatalogues 66

\section{CONCLUSIONES}

Nuestras con clu sio nes gi ran prin cipal men te en tor no a las me di das que se to ma ron para adop tarla propues ta de Jua na Man riquedeLaraenla for mación de biblioteca rios en México, aun que se in clu ye tam bién una re fle xión so brela impor tan cia que en mate ria de democra tiza ción debiblio te cas ydellibroten dría el he cho dequelas bibliotecaspúblicasmexicanascontaranconpersonalprofesionalenbibliotecología.

Cabeseñalarque aunquela suge rencia de forma ción he chaporJuana fuevalorada en su mo men to por To rres Bo det, éste sa lió delDe par ta men to deBiblio te cas de la SEP ante el cam bio de go bierno y no pudo po nerla en prác ti ca sino has ta 1945, cuando como Secretario de Educación Pública tuvo la oportunidad de abrir la Escuela NacionaldeBibliotecariosy Archivistas.

No obstante lo anteriormente mencionado conviene señalar que la propuesta de forma ciónhe cha porJua na Man rique deLarase adop tó de in media to en 1925, al fundarse en el periodo de gobierno de Plutarco Elías Calles con Esperanza VelázquezBringas alacabezadelDepartamentodeBibliotecas, lasegundaEscuelade Bibliotecarios.

Estase gun da es cuela de biblio te ca rios se creó conelapo yo de Velás quez Brin gas ygra ciasalimpul so quele die ra al proyectola re cién crea da Aso cia ción deBibliote carios Mexica nos. Cabe aco tarque VelázquezBringas, al igual quelos cons titu cionalistas, Man rique deLarayla re cien te aso cia ción debiblio te carios, de fen die ronlanecesidad de formarpersonalparalas biblio te cas a par tirdela fun da ción de una escuela d e biblioteconomía.

65 EngydopediadLibaryandInformatiónSäencee v. 26, New York, Mar cel Dekker, 1978, pp. 322-330. Ernest AlbertSa va ge (1977-1966) for mó par te del gru po de biblio te ca rios que erigie ron es tán daresbiblioteconómicos paraelserviciodelas bibliotecaspúblicas británicas delsiglo XX.Defendió la idea de que las biblio te cas pú blicas de las ciu da des de Gran Bre ta ña es ta ble cie ran serviciospara la in dus tria lo cal y sus fir mas co mer cia les. A fin de con tri buir más a re sol ver los proble mas oca sionadosporlaguerra recomen dó elestablecimientodela NationalLen dingofScientific and Te chnical Literature. Y en general buscó que las bibliotecas públicas proporcionaran servicios de alta calidad para cons ti tuirlas en verda de ros cen tros cul tu rales dela co mu nidad. Tam bién derivó del modelo Americanosuinterésenladeparta mentaliza ción pormateria. Alrespectoescribióellibro SpeaalLibranianshipingenerallibraies Eigual men te de este mo de lo re to móla in fluen cia de la aso cia ción de biblio te ca rios en el de sa rrollo del sis te ma biblio te ca rio, en es pecial paralas bi blio te cas públicas. Jugó un im por tan te pa pel en la me jo ría de la Li brary Asso cia tion. Des pués de su retiro,en 1942, continuó escribiendo y el mejor de sus libros de esa época es A Librananlodksatreades

66 Juana Manrique de Lara, "Anotaciones y revistas de libros", en Op Cit, p. 89. 
Entendemos que Velázquez Bringas, al igual que Manrique de Lara, comprendía que la es cueladebía formartan to para orga nizartécnica men telas bibliotecas como para prestarmejo res serviciosalpúblico.La escuela fundada en 1925adop tó enesencia el plan de estudios que Juana, convencida de los avances biblioteconómicos logrados en Es ta dos Unidos, ${ }^{67}$ le ha bía pro pues to des de Nue va York a To rres Bo det. Esteplan am pliabala visión dela for mación técnica ${ }^{68}$ y elám bito de competen cia del bibliote cario al plan tearlanece sidadde in troducirla ma teria de selec ción. El programa planteaba también la necesidad de incluir la asignatura de consulta como parte im por tan te de la for ma ción para el ser vi cio al pú blico e in tro du cía como par te dela formación de los bibliotecariosmaterias o conferencias para mejorar su formación enculturageneral. ${ }^{69}$

La con cep ción de la pro pues ta he cha por Jua na per meó no sólo el plan de es tudios de la es cue la de 1925 y la que de fi ni tiva men te se abrie ra en 1945 (Es cue la Nacional de Bibliotecarios y Archivistas) sino también los cursos que surgieron después de 1925 y las escuelas que nacieron después de la Escuela Nacional de BibliotecariosyArchivistas.

Noobstante,lalentaperocrecienteexistenciadeprofesionales delabiblio tecología ylosbajossala riospaga dos enlasbiblio tecaspúblicas nohan permitidoqueelbibliotecario profesional se desempeñe en ellas, situación que a nuestro juicio contribuiría a mejorarestefundamental tipodebiblioteca,ya cumplirsupapel social, aho ra favorecido,almenosteóricamente,porlaexistenciadelastecnologías deinformación.

67 Es tela Mo ra les Cam pos. EducadónbibliotecolóġaenMéxiø1915-1954 México, Cen troUniversita riodeInvestigacionesBibliotecológicas-UNAM, 1988,p. 8. La in fluen cia de Man ri que de Lara en elcita do plan dees tu dios tam bién es re co no cida por Mo rales cuan do señala. "En esteplan de es tudios de 1925 ya en con tra mos un cur so de Re fe ren cia que nos per mi te pen sar que no nada más se preocupaban por la or ga ni za ción y la bi blio te ca, sino del usua rio de ésta; y la in fluencia de Jua na Manrique de Lara, que ya había tomado este curso en los Estados Unidos".

68 "Plan de Es tu dios de la Es cue la Na cio nal de Biblio te ca rios", en BoletíndelaA sodaacóndeBiblioteca nosMexicanos, I, 3 (15 fe bre ro, 1925), pp.46-48. El plan de es tu dios com pren día ade más de la ma teria de Bibliografía y Trabajos de Referencia, la de Selección de Libros. La inclusión de estas materias habla de que se retomaba la concepción ampliada de formación técnica propuesta por Jua na. Man rique de Lara fue in vi ta da para di se ñar al lado de Juan B. Iguíniz el plan de es tu dios y fue laprofe so radelas dos ma terias an tes cita das. Cabe señalarque seimpartieron tambiénlas materias de: bibliología, cla si fica ción, ca taloga ción y orga niza ción debiblio te cas y queade más de Iguí nizy Juanafiguraron como pro feso res otras importan tes perso nalida des dela pro fe sión.

69 J. M. Puig Casau ranc. LaøucadónpúldicaenMéxicoatravésdelosmensajespresidendalesdesdlaconsuma dóndelaindependandahastanuestrosdías Mé xi co, Se cre ta ría de Edu ca ción Pú blica, 1926,p.298. Este plan se complemen ta ba conal terna tivas para mejo rar la cul tu ragene raldel biblio tecario, en foque que como he mos vis to ya ha bía sido su ge ri do Man rique de Lara, de ma ne ra que se dic ta ron "dos o tres con fe ren cias so bre arte, cien cias y li te ra tu ra, con obje to de que los biblio te ca rios y los nue vos es tudian tes debiblioteca rios, va yan am plian do sucul tura." 


\section{O BRAS CON SULT AD AS}

\section{Libros}

DiariodedebatesddCongresoConstituyente1916-1917.2 v... México, Ins ti tu to Nacional de Estudios Históricos de la Revolución Mexicana, 1985.

Engdqpedia of Library and Infomation Säence, 35 v. New York, Marcel Dekker, 1978.

León, Ni co lás,Biblioteconomáa;notasdlasleccionesaralesdlProfesorDr.Nicolás Leén enla Esauda Nacional deBiblicteaniosy Archiveros México, Antigua Imprenta de Murguía, 1918, 167 pp.

Morales Campos, Estela, Eduraaón biblictedóóga en Méxic 1915-1954 México, Centro Universitario de Investigaciones Bibliotecológicas, UNAM, 1988, 112 pp.

Puig Casau ranc, J: M. LaeducadónpúblicaenMéxi œatravésdelosmensajespre sidenaalesdesdela consumacóndelaindependenaiahastanuestrosdías Mé xico, Secretaría de Educación Pública, 1926, 505 pp.

Vázquez Mantecón, Carmen, Æ. al., Lasbiblictecasmexicamasend sigoXIX. México,SecretaríadeEducaciónPública-Dirección GeneraldeBibliotecas, $1987,253 \mathrm{pp}$.

\section{Artículos}

“Aviso”, en El libroyd pudblo I, 2 (abril, 1922), p. 16.

Fernández de Zamora, Rosa María. "Cronología bibliotecaria mexicana 1900-1988”, en InvestigaiónBiblideedógica II, 3 (julio-diciembre, 1987), pp. $48-59$.

García Núñez, Luz. "Memoria de la conferencia de la American Library Asso cia tion y la South west Li brary Asso cia tion", en BoletíndelaSecreta núa deEduradónPública, I, 4, (1er. Semestre, 1923), pp.278-285.

Iguíniz,Juan B. "Apun tes para la his to ria de la en se ñan za de la biblio te cono mía en Mé xi co", enBoletíndelaEscudaNadionaldeBibliotecaniosyAndi vistasIII, 3-4 (enero.abril,1954), pp. 13-17.

- - "Cla ses de biblio lo gía y biblio te co no mía", en BoletíndelaSecetanáade Eduraaón Púdica, I, 4, (1er semestre, 1923), pp. 295-296.

"Informe leído del Departamento en el Congreso de Bibliotecarios, de Austin Te xas", en Bdéńndela SecutańadeEducaaónPúdica, I, 3, (enero, 1923), pp 334-340. 
"In formeque rin delaSeño rita Es peranza Velázquez Bringas, Jefe delDepar ta men to de Biblio te cas al Doc tor José Ma nuel Ruiz Casau ranc, Secretario de Educación Pública, acerca de las labores desarrolladas durante el mes de enero", en Bdéíndela Seretaria deEduradónPública, III, 9 (febrero, 1925), pp.137-142.

"In vi ta ción al pue bloy a la pren sa", en Ellibroyd puedloI, 1 (mar zo, 1922), p. 1.

Loe ra y Chá vez, Agus tín. "La pri me ra Es cue la N. de Bi blio te ca rios y Archiveros”, en BoletíndelaBibliotecaNacionaldeMéxica, II, 3 (ene ro, 1916), pp.121-123.

Man rique deLara,Juana. “Ano ta ciones y revis tas delibros", en Ellibroye puedloIII, 4-6 (abril-junio, 1924), pp. 89-92.

--. "Biblio te cas in fan tiles y ju ve niles", en Biblosiv, 158- 159 (28 ene ro, 4 febrero, 1922), p.15, 18-19.

- -. "Bibliotecas y bibliotecarios", en El libroyd Puedld' III, 1-3 (eneromarzo, 1924), pp. 33-35.

--. "La selec ción de li bros para las di fe ren tes cla ses de bi blio te cas y en es pe cial para las biblio te cas públicas”, en Ellibroyd pueblo,III, 10- 12 (octubre- diciembre, 1924), pp. 227-229.

--. "Progra ma dela Es cuela deBiblio te ca rios ane xa a la Biblio te ca Pública de la ciu dad de Nue va York", en Ellibroyd pueblo II, 8-10 (oc tu brediciembre, 1923), pp. 201-202.

- - " "Proyecto de reformas e introducción de sistemas de biblioteconomía, se gún los mé to dos Nor te Ame ri ca nos, en las bi blio te cas dela Repúblicamexicana" enEllibroyd puedlo, III,7-9 (julio-septiembre,1924), pp. 173-175.

"Plan de Estudios de la Escuela NacionaldeBibliotecarios", en Boletínde laAsociacióndeBibliotecariosMexicanos, I, 3 (15 fe bre ro, 1925), pp.46- 48.

"Requisitos para ser bibliotecario", en El Libroy d Pudblo I, 6 (agosto, 1922), p.47.

To rre de Ote ro, Ma ría Lui sa de la. "El Alma de la bi blio te ca", en ElLibroy d Pudblo, II, 2-3 (abril-mayo, 1923), pp.45-47.

Vasconcelos, José. "Reglamento de las Bibliotecas Públicas: disposiciones generales”, en El Libroyd Puddo, I, 4 (junio, 1922), p. 26. 


\section{Tesis}

Añorve Guillén, Martha Alicia. El despetar dela vocación bibiideeconónica de Juana ManniquedeLara(1897-1922)endmarcodelasinstitucionesbiblioteca nasdesutiempa(Tesis demaes tría en Biblio te cología)México,División de Estudios de Posgrado, Facultad de Filosofía y Letras, Universidad Nacional Autónoma de México, 2002, 306 p.

\section{Documentosdearchivo}

NNC-RBML. The Columbia University Rare Books \& Manuscript Library. Library School of the New York Public Library- Box 31 Juana Manrique de Lara 1923-1924. [Student record].

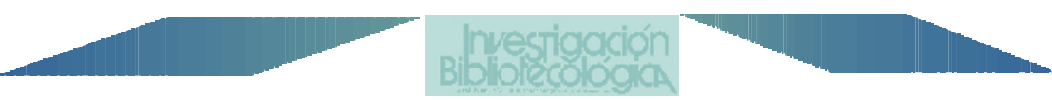

